\title{
HIF-1 $\alpha$ attenuates neuronal apoptosis by upregulating EPO expression following cerebral ischemia-reperfusion injury in a rat MCAO model
}

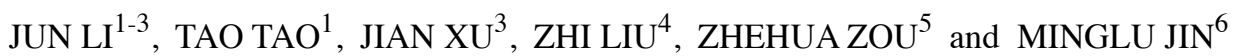 \\ ${ }^{1}$ Department of Rehabilitation Medicine, Guizhou Provincial People's Hospital, Guiyang, Guizhou 550002; \\ ${ }^{2}$ Department of Neurology, Wuhan Fourth Hospital, Wuhan, Hubei 430000; Departments of ${ }^{3}$ Neurology and ${ }^{4}$ Pharmacy, \\ The Affiliated Hospital Guizhou Medical University, Guiyang, Guizhou 550001; ${ }^{5}$ Department of General Practice, \\ The First Hospital of Qinhuangdao, Qinhuangdao, Hebei 066000; ${ }^{6}$ Department of Neurology, Qijiang Hospital of \\ The First Affiliated Hospital of Chongqing Medical University, Chongqing 404100, P.R. China
}

Received August 2, 2019; Accepted December 30, 2019

DOI: $10.3892 / \mathrm{ijmm} .2020 .4480$

\begin{abstract}
Hypoxia-inducible factor- $1 \alpha(\mathrm{HIF}-1 \alpha)$ is a key transcriptional factor in response to hypoxia and is involved in ischemic stroke. In the present study, the potential for HIF-1 $\alpha$ to inhibit neuronal apoptosis through upregulating erythropoietin (EPO) was investigated in a transient middle cerebral artery occlusion (tMCAO) rat stroke model. For this purpose, a recombinant adenovirus expressing HIF-1 $\alpha$ was engineered (Ad-HIF-1 $\alpha$ ). Control adenovirus (Ad group), Ad-HIF-1 $\alpha$ (Ad-HIF-1 $\alpha$ group) or Ad-HIF-1 $\alpha$ in addition to erythropoietin mimetic peptide-9 (EMP9), an EPO-receptor (-R) antagonist (Ad-HIF-1 $\alpha+$ EMP9 group), were used for an intracranial injection into rat ischemic penumbra $1 \mathrm{~h}$ following MCAO. All rats demonstrated functional improvement following tMCAO, while the improvement rate was faster in rats treated by Ad-HIF-1 $\alpha$ compared with all other groups. The EPO-R inhibitor partially reversed the benefits of Ad-HIF-1 $\alpha$. Apoptosis induced by tMCAO was significantly inhibited by Ad-HIF-1 $\alpha(\mathrm{P}<0.05)$. The expression of HIF-1 $\alpha$, evaluated by immunohistochemistry either in neurons or astrocytes, was upregulated by Ad-HIF-1 $\alpha$. Both EPO mRNA and protein expression were increased by Ad-HIF-1 $\alpha$, however, there was no significant change of EPO-R either on an mRNA level or protein level. Furthermore, EMP9 did not change the EPO expression which was upregulated by Ad-HIF-1 $\alpha$. Activated caspase 3 in neurons was suppressed by Ad-HIF-1 $\alpha$. Activated caspase 3 downregulated by HIF-1 $\alpha$ was partially blocked by EMP9. Altogether, the present data demonstrated that HIF-1 $\alpha$
\end{abstract}

Correspondence to: Dr Tao Tao, Department of Rehabilitation Medicine, Guizhou Provincial People's Hospital, 83 Zhong Shan East Road, Nanming, Guiyang, Guizhou 550002, P.R. China

E-mail: 13985162824@163.com

Key words: hypoxia-inducible factor- $1 \alpha$, cerebral ischemia, erythropoietin, apoptosis, caspase 3 attenuates neuronal apoptosis partially through upregulating EPO following cerebral ischemia in rat. Thus, upregulating HIF-1 $\alpha$ subsequent to a stroke may be a potential treatment for ischemic stroke.

\section{Introduction}

Stroke, following coronary heart disease and cancer, is the third most common cause of mortality in numerous countries (1), and it has become an enormous health issue globally (2). In China, stroke is one of the most common causes of mortality and long-term disability in previous years $(3,4)$. More than 10 million Chinese individuals are living with stroke, with 2.4 million novel cases reported in 2013 (5). Acute ischemic stroke is the most common type of stroke, which accounts for $60-80 \%$ of all cases (6). To date, antiplatelet agents and thrombolytic medications are the only drug treatment options of ischemic strokes that are supported by strong clinical evidence (7). However, the applications of these two treatments are often limited by the potential risk of cerebral bleeding and a narrow treatment time window. The percentage of patients with ischemic stroke who are receiving thrombolytic therapy is no more than $3.5 \%$ in the United States (8) and only 1 to $3 \%$ in China (9). Overall, there remains a very limited number of effective interventions for ischemic stroke following decades of practice and investigation. Therefore, it is necessary to further investigate feasible and effective treatment options.

Hypoxia-inducible factor- $1 \alpha(\mathrm{HIF}-1 \alpha)$ serves an important function in the cellular adaptation to hypoxia (10). HIF-1 $\alpha$ is tightly regulated by oxygen tension (11). Under normoxia, HIF- $1 \alpha$ is ubiquitinated and degraded by prolyl-hydroxylases (12). Under hypoxic conditions, HIF-1 $\alpha$ is stabilized and translocated to the nucleus where it dimerizes with HIF-1 $\beta$, the other subunit of HIF-1, and activates the transcription of its target genes involved in survival in hypoxia (11). HIF-1 is able to induce the transcription of erythropoietin (EPO) and vascular endothelial growth factor (VEGF) (13). These responses increase oxygen supply to oxygen-deprived tissues by promoting erythropoiesis and angiogenesis. A continuous 
supply of oxygen to the central nervous system (CNS) is extremely important for maintaining normal brain function. It is vital that neurons in the CNS are able to detect and respond rapidly to brain hypoxia (13). It has been reported that hypoxic preconditioning increases tolerance to hypoxic-ischemic-induced brain injury and decreases apoptosis by upregulating the expression of HIF-1 $\alpha$ (14). Furthermore, hypoxic preconditioning in transplanted bone marrow stromal cells promoted their capability of regeneration and therapeutic potential to treat rat ischemic stroke (15).

EPO, a target gene of HIF-1, is a glycoprotein hormone that consists of a single polypeptide consisting of 166 amino acids folded into four $\alpha$-helices (16). EPO production was revealed to be induced by hypoxia in a murine brain, uterus and kidney (17). EPO administration protected embryonic neurons and postnatal hippocampal neurons against cell death induced by hypoxia (18). In neonatal rats, delayed EPO administration stimulated oligodendrogenesis and attenuated white matter damage in hypoxic/ischemic brain injury (19). Furthermore, EPO attenuated inflammatory response by decreasing cyclooxygenase 2 and inducible nitric oxide synthase, suppressing microglial activation and inhibiting autophagy activation in burn-induced motor neuron neuroinflammation (20).

However, to the best of our knowledge, there are no reports available concerning the effect of exogenous HIF-1 $\alpha$ in ischemic stroke models at present. Furthermore, there are also no reports to the best of our knowledge concerning the effects of EPO induced by exogenous HIF-1 $\alpha$ on neuronal apoptosis following cerebral ischemic stroke.

In the present study, a recombinant adenovirus engineered to express HIF-1 $\alpha$ (Ad-HIF-1 $\alpha$ ) was used to treat a transient middle cerebral artery occlusion (tMCAO) rat model. The aim of the present study was to investigate whether HIF-1 $\alpha$ may decrease neuronal apoptosis by inducing EPO.

\section{Materials and methods}

Ethics. The present study followed all guidelines stated in Guide for the Care and Use of Laboratory Animals, prepared by the Committee on Care and Use of Laboratory Animals of the Institute of Laboratory Animal Resources Commission on Life Sciences (National Research Council, China; 1996). The animal studies were ethically approved by the local Animal Experimentation Ethics Committee (Guizhou Provincial People's Hospital, Guizhou, China) for animal experimentation. All efforts were made to minimize the number of rats used and their suffering.

Transient focal cerebral ischemia rat model. A rat model was produced as previously described (21). A total of 32 male Sprague-Dawley rats weighing 230-250 g purchased from Guizhou Medical University were used in this study. Rats were housed individually and had ad libitum access to food and water. The temperature in the room was $25^{\circ} \mathrm{C}$ and the room was under the condition of a 12-h light-dark cycle. Rats were initially anesthetized with $0.05 \mathrm{mg} / \mathrm{g}$ ketamine followed by administration of $0.01 \mathrm{mg} / \mathrm{g}$ xylazine intraperitoneally. Oxygen was supplied through a face mask during surgery. tMCAO was induced by following the method of intraluminal vascular occlusion. Briefly, the right external carotid artery
(ECA), common carotid artery and internal carotid artery (ICA) were isolated. A 4-0 nylon suture with a $3 \mathrm{~cm}$ length and a slightly enlarged and rounded tip was inserted from the ECA into the lumen of the ICA to block the origin of the MCA. The distance was 18.5 to $19.5 \mathrm{~mm}$ from the tip of the suture to the bifurcation of the common carotid artery. Following $60 \mathrm{~min}$ of MCAO, reperfusion was performed by withdrawal of the suture until the tip cleared the lumen of the ECA. In the sham group, the rats underwent identical procedures without the insertion of the nylon monofilament.

Treatments for rats. Rats were treated as previously described $(22,23)$. A recombinant adenovirus carrying the HIF-1 $\alpha$ gene and the green fluorescent protein gene (Ad-HIF-1 $\alpha$ ) was constructed using the AdEasy ${ }^{\circledR}$ System (American Type Culture Collection). Previous studies have reported that the recombinant adenovirus is able to stably express HIF-1 $\alpha$ in neural stem cells and differentiated derivatives $(24,25)$.

At $1 \mathrm{~h}$ subsequent to $\mathrm{MCAO}$, the rats were randomly divided into four groups $(n=8)$ : Sham group, ischemia+Ad (Ad group), ischemia+Ad-HIF-1 $\alpha$ (Ad-HIF-1 $\alpha$ group) and ischemia+Ad-HIF-1 $\alpha+$ erythropoietin mimetic peptide-9 (EMP-9) (Ad-HIF-1 $\alpha+$ EMP-9 group). Animals were anesthetized with equithesin ( $3 \mathrm{ml} / \mathrm{kg}$ administered intraperitoneally) and transferred to a stereotaxic apparatus. A 2 to $5 \mathrm{~mm}$ incision was created in the scalp, $1.5 \mathrm{~mm}$ lateral to the bregma, under an aseptic technique. By using a dental drill, a burr hole was produced in the bone $3 \mathrm{~mm}$ lateral to bregma. Approximately $10 \mu \mathrm{l} \mathrm{Ad}, 10 \mu \mathrm{l}$ Ad-HIF-1 $\alpha$ or $10 \mu \mathrm{l}$ Ad-HIF-1 $\alpha$ were slowly injected into the ischemic area at a depth of $2.0 \mathrm{~mm}$ from the surface of the brain over $20 \mathrm{~min}$. Prior to retraction, the needle was maintained in the brain for an additional $5 \mathrm{~min}$. To inhibit EPO-receptor (-R) functions, rats in the Ad-HIF-1 $\alpha+$ EMP-9 group received an intraperitoneal injection of EMP-9 (cat. no. MBS8243539; MyBioSource, Inc.), a proven EPO-R antagonist, at $1.0 \mathrm{mg} /$ time, four times at $1 \mathrm{~h}$ intervals per day from day 1 to day 7 following tMCAO. The EMP-9 was dissolved in $1 \mathrm{X}$ phosphate buffered saline (PBS) at a final concentration of $1 \mathrm{mg} / \mathrm{ml}$. Rats in the other two groups (the $\mathrm{Ad}$ group and Ad-HIF-1 $\alpha$ group) received equal volume injections of PBS. Body temperature during surgery was controlled with a thermostatically regulated heating pad at $37.0 \pm 0.5^{\circ} \mathrm{C}$ and was monitored by a rectal thermometer. Rats were returned to their cages on the warm pad and allowed to recover from anesthesia following surgery. Agar chow instead of solid chow was used as the rats experienced hemiplegia and hemidysesthesia following MCAO. Humane endpoints of the study included major changes in body weight, external physical appearance, behavior and physiological measures (e.g., body temperature, hormonal fluctuations, clinical pathology). A total of three rats were lost to intracerebral hemorrhage four rats were lost to fever. Extra rat(s) were added to the groups where necessary to ensure 8 rats/group.

Behavioral testing. A modified neurological severity score (NSS) assessment was performed on days $0,1,3,5$ and 7 following tMCAO by a well-trained researcher who was blinded to the study conditions. The NSS assessment consisted of motor (muscle status and abnormal movement), sensory 
(visual, tactile and proprioceptive), reflex and balance tests as described previously (26). Neurological function was graded on a scale of 0 to 18 (normal score, 0 ; maximum deficit score, 18). A higher score indicated a more severe injury.

Histological analysis. On day 7 following ischemia, four rats from each group were sacrificed under deep anesthesia and transcardially perfused using $1 \mathrm{X}$ PBS followed by $4 \%$ paraformaldehyde for histological analysis. The injection site was located on the brain and $3 \mathrm{~mm}$ of the frontal and dorsal sides of the injection site $(6 \mathrm{~mm}$ thickness in total) were isolated. The isolated regions were cryoprotected with $30 \%$ sucrose for $24 \mathrm{~h}$ following $24 \mathrm{~h}$ fixation at $4^{\circ} \mathrm{C}$ in $4 \%$ paraformaldehyde. To evaluate HIF-1 $\alpha$ expression, serial $10 \mu \mathrm{m}$-thick coronal sections were processed. Sections were blocked by $3 \% \mathrm{BSA}$ at room temperature for $30 \mathrm{~min}$ and stained with mouse anti-HIF1 $\alpha$ (1:200; cat. no. NB100-479; Novus Biologicals, LLC), HIF-1 $\alpha$ (1:200)/RNA binding fox-1 homolog 3 (NeuN; 1:500; cat. no. ABN90; EMD Millipore) and HIF-1 $\alpha$ (1:200)/glial fibrillary acidic protein (GFAP; 1:500; cat. no. sc-33673; Santa Cruz Biotechnology, Inc.) antibodies to identify whether neurons or astrocytes expressing $\mathrm{HIF}-1 \alpha$ at $4^{\circ} \mathrm{C}$ for overnight. Immunohistochemistry for activated caspase 3 was performed using an anti-active caspase 3 antibody (1:100; cat. no. ab2302; Abcam) and active caspase 3 (1:50)/NeuN (1:500; cat. no. ABN90; EMD Millipore) at $4^{\circ} \mathrm{C}$ for overnight. The sections were incubated with goat anti-rabbit biotin-conjugated secondary antibody (1:400; cat. no. sc-2040; Santa Cruz Biotechnology, Inc.) or donkey anti-rabbit Alexa Fluor 488 (1:400; A21206; Invitrogen; Thermo Fisher Scientific, Inc.)/goat anti-mouse Alexa Fluor 594 (1:400; A11005; Invitrogen; Thermo Fisher Scientific, Inc.) at room temperature for $1 \mathrm{~h}$. Images were captured using an Olympus BX41 light microscope (magnification, x400; Olympus Corporation) for HIF-1 $\alpha$ and active caspase 3 , and an Olympus BX51 Fluorescence microscope (magnification, x400; Olympus Corporation) for immunofluorescence staining. Image J software 1.46 (National Institute of Health) was used to quantitate the levels of immunoreactivity.

TUNEL assay. To determine the number of apoptotic cells, a TUNEL assay was performed using an In Situ Cell Death Detection kit (cat. no. 11684795910; fluorescein; Roche Diagnostics) according to the manufacturer's protocol. Briefly, slides with brain tissue were washed three times for $5 \mathrm{~min}$ with PBS and permeabilized with $0.5 \%$ Triton X-100 in PBS for $10 \mathrm{~min}$. Then, tissues were incubated in $100 \mu \mathrm{l}$ TUNEL reaction mixture for $1 \mathrm{~h}$ at $37^{\circ} \mathrm{C}$ in a chamber with a humidified atmosphere. As a positive control, tissues were treated with RNase-free DNase I (400 U/ml; Qiagen, Inc.) at room temperature for $15 \mathrm{~min}$ prior to incubation with the TUNEL reagent. Tissues were incubated with the TUNEL reagent in the absence of terminal deoxynucleotidyl transferase as a negative control for the experiment. Subsequent to washing three times with PBS, the slides were mounted with a mounting medium containing 4,6-diamidino-2-phenylindole (DAPI). Images were captured using an Olympus BX51 Fluorescence Microscope (magnification, $\mathrm{x} 200$ ). TUNEL positive cells were calculated by counting the positively stained cells in each of the five fields of vision.
Reverse transcription-quantitative PCR (RT-qPCR). On day 7 following $\mathrm{MCAO}$, three rats from each group were sacrificed to collect fresh samples. The penumbral cortex (around the injection site, $\sim 5 \mathrm{~mm}$ in diameter) on the ischemic side was dissected and maintained in liquid nitrogen for RT-qPCR and western blotting. Total RNA was extracted from penumbral cortex by using the RNeasy kit (Invitrogen; Thermo Fisher Scientific, Inc.) according to the manufacturer's protocol. Using a PrimeScript RT Reagent kit (Takara Biotechnology Co., Ltd.), RNA samples were subjected to reverse transcription at $37^{\circ} \mathrm{C}$ for $60 \mathrm{~min}$. For each rat, the reactions were run in triplicate in three independent experiments. The FAM-labeled fluorophore was obtained from TaqMan ${ }^{\circledR}$ (Thermo Fisher Scientific, Inc), and the qPCR thermocycling conditions were as follows: $93^{\circ} \mathrm{C}$ for $3 \mathrm{~min}$, followed by 40 cycles of $93^{\circ} \mathrm{C}$ for $1 \mathrm{~min}, 55^{\circ} \mathrm{C}$ for $1 \mathrm{~min}$ and $72^{\circ} \mathrm{C} 1 \mathrm{~min}$. Rotor-Gene software v2.3 (Qiagen) accompanying the Applied Biosystems ${ }^{\mathrm{TM}} 7500$ Real-Time PCR System (Applied Biosystems; Thermo Fisher Scientific, Inc.) was used to collect and analyze data. The $\mathrm{Cq}$ values of each sample were normalized to the corresponding GAPDH Cq values, and relative expression levels were calculated using the $\Delta \Delta \mathrm{Cq}$ method (27). The primers used were as follows: EPO forward, 5'-CATCTGCGACAGTCGAGTTCTG-3' and reverse, 5'-CACAACCCATCGTGACATTTTC-3'; EPO-R forward, 5'-ACACGTCGAGTTTTGTGCCA-3' and reverse, 5'-TGATGATGCGGTGGTAGC-3'; GAPDH forward, 5'-CAT GGCCTTCCGTGTTCCTA3 and reverse, 5'-TACTTGGCA GGTTTCTCCAGG3.

Western blot assay. Brain tissues were homogenized in RIPA lysis buffer (Beijing BLKW Biotechnology Co., Ltd.), followed by centrifugation at $10,000 \mathrm{x}$ g for $10 \mathrm{~min}$ at $4{ }^{\circ} \mathrm{C}$. BCA method was used for quantitative analysis the protein samples. Proteins $(30 \mu \mathrm{g})$ from brain tissues were separated by $10 \%$ SDS-PAGE under reducing conditions. Subsequently, the proteins were transferred to nitrocellulose membranes. A total of 3\% milk in PBS with $0.05 \%$ Tween-20 (PBST) was used to block the unspecific binding of antibodies at room temperature for $1 \mathrm{~h}$. Then, the blots were incubated with the following primary antibodies: Mouse Anti-HIF1 $\alpha$ (1:10,000; cat. no. NB100-479; Novus Biologicals, LLC), anti-EPO (1:1,000; cat. no. sc-5290; Santa Cruz Biotechnology, Inc.), anti-EPO-R (1:1,000; cat. no. sc-365662; Santa Cruz Biotechnology, Inc.) or mouse anti- $\beta$-actin antibody (AC-15; 1:3,000; cat. no. sc-69879; Santa Cruz Biotechnology, Inc.) overnight at $4^{\circ} \mathrm{C}$. Blots were then incubated with a rabbit anti-goat IgG-HRP (1:3,000; cat. no. sc-2922; Santa Cruz Biotechnology, Inc.) or goat anti-mouse IgG-HRP (1:3,000; sc-2005; Santa Cruz Biotechnology, Inc.) for $1 \mathrm{~h}$ at room temperature and washed three times with PBST. An enhanced chemiluminescence (ECL) western blotting detection reagent (cat. no. RPN2232; GE Healthcare) was used for visualization. An Odyssey Infrared Imaging system and software (model 9120; LI-COR Biosciences) were used to evaluate the specific signals and the corresponding band intensities.

Statistical analysis. Data are presented as the mean \pm standard deviation and were analyzed using one-way analysis of variance followed by a Bonferroni post hoc-test using SPSS 15.0 


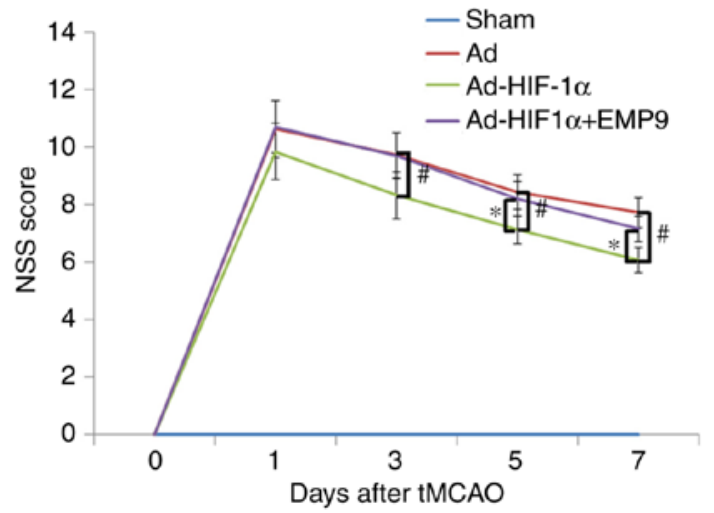

Figure 1. Ad-HIF-1 $\alpha$ treatment promoted recovery in rats with tMCAO. An NSS score was determined on day $0,1,3,5$ and 7 following treatment. No neurological deficit was observed in the Sham group. Compared with the Ad-treated rats, Ad-HIF-1 $\alpha$-treated rats had significantly fewer severe symptoms from day 3 following treatment. ${ }^{~} \mathrm{P}<0.05$ vs. Ad-treated rats. Compared with Ad-HIF-1 $\alpha+$ EMP9-treated rats, Ad-HIF-1 $\alpha$-treated rats also exhibited significantly better recovery from day 3 onwards. $\mathrm{P}<0.05$ vs. Ad-HIF-1 $\alpha$ +EMP9-treated rats. Ad-HIF-1 $\alpha+$ EMP9-treated rats did not recover better compared with Ad-treated rats within 7 days. NSS, neurological severity score; tMCAO, transient middle cerebral artery occlusion; Ad, adenovirus; HIF-1 $\alpha$, hypoxia-inducible factor-1 $\alpha$; EMP9, erythropoietin mimetic peptide-9.

software (SPSS, Inc.). P $<0.05$ was considered to indicate a statistically significant difference.

\section{Results}

Ad-HIF-1 $\alpha$ treatment promotes neurological functional recovery in $t M C A O$ rats. To investigate if treatment with Ad-HIF-1 $\alpha$ may improve sensorimotor deficit, the present study compared NSS scores. The score of all rats in all groups was 0 prior to tMCAO (day 0). No neurological functional deficits were revealed in the Sham group. Following tMCAO on day 1 , there were no significant differences among the three groups in terms of NSS scores. Neurological functional deficits evaluated by the NSS assessment revealed a progressive recovery in each group from day 3 to 7 following tMCAO. Ad-HIF1- $\alpha$ treatment demonstrated a significantly better improvement in NSS scores compared with the Ad group on post-ischemia day 3, 5 and 7 ( $\mathrm{P}<0.05$; Fig. 1). EMP9 partially abolished the benefits of Ad-HIF1- $\alpha$ treatment (Fig. 1). Body weights among the 4 groups were not significantly different across the 7 days (data not shown).

Ad-HIF-1 $\alpha$ attenuates apoptosis in tMCAO rats. To evaluate whether HIF-1 $\alpha$ may decrease the number of apoptotic cells in rats with tMCAO, the present study treated rats $1 \mathrm{~h}$ following tMCAO with Ad, Ad-HIF-1 $\alpha$ or Ad-HIF-1 $\alpha$ and EMP9. Very few TUNEL-positive apoptotic cells were detected (Fig. 2A), compared with the total number of cells (Fig. 2B), as observed through DAPI staining, in the Sham group. However, rats in the Ad group had a substantially greater number of apoptotic cells in the pre-ischemic area on day 7 following tMCAO (Fig. 2C and D). HIF-1 $\alpha$-treated rats had substantially fewer apoptotic cells on day 7 compared with the Ad-treated rats (Fig. 2E and F). In the Ad-HIF1- $\alpha$ with EMP9-treated rats, the number of apoptotic cells remained high (Fig. $2 \mathrm{G}$ and H). Compared with rats treated with Ad and Ad-HIF1- $\alpha$ with
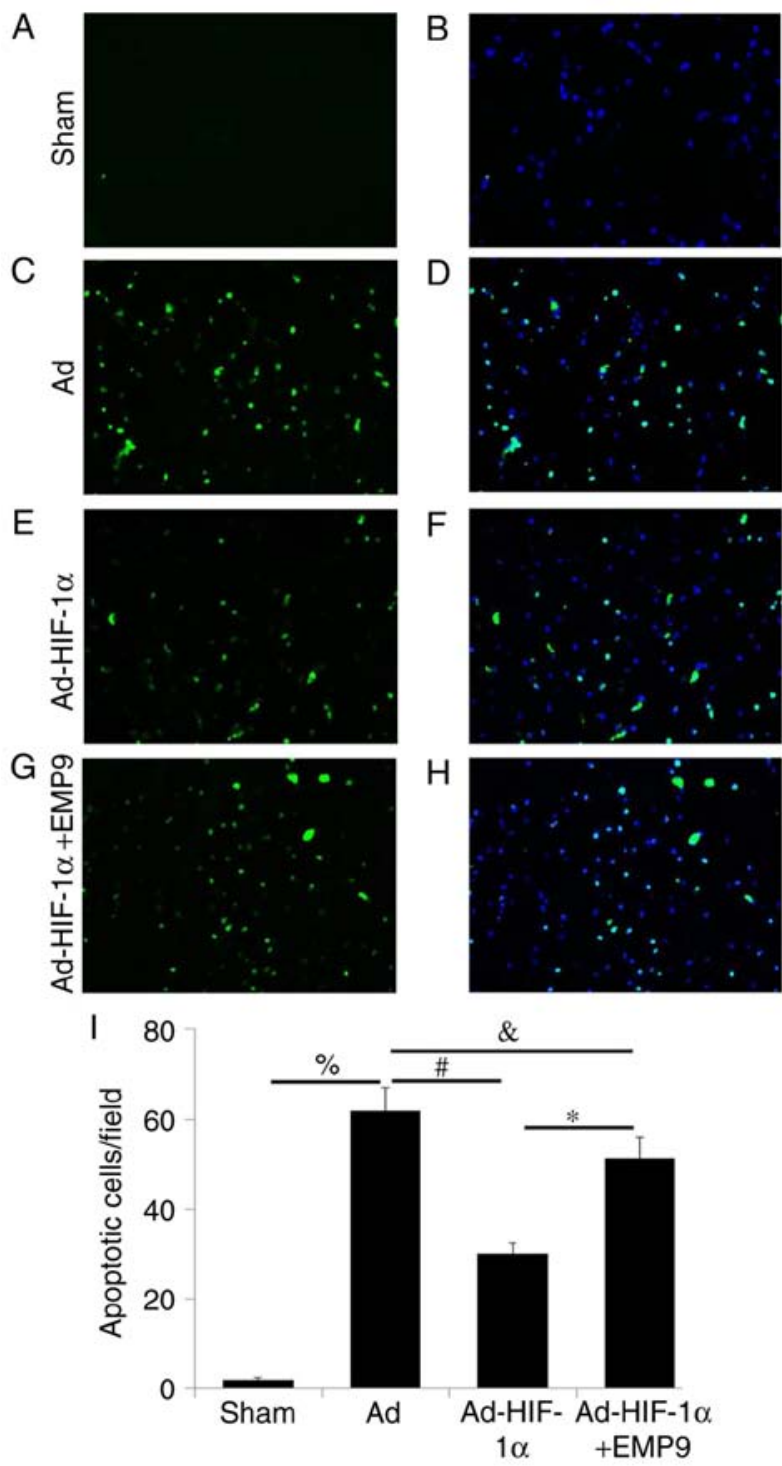

Figure 2. Ad-HIF-1 $\alpha$ reduced apoptosis following tMCAO. Apoptotic cells were determined by TUNEL staining. (A) Apoptotic and (B) total cells detected in the Sham group. (C) Apoptotic and (D) total cells detected in the Ad-treated group. (E) Apoptotic and (F) total cells detected in the Ad-HIF-1 $\alpha$-treated group. $(\mathrm{G})$ Apoptotic and $(\mathrm{H})$ total cells detected in the Ad-HIF-1 $\alpha+$ EMP9-treated group. (I) Quantified number of apoptotic cells. ${ }^{*} \mathrm{P}<0.0,{ }^{\#} \mathrm{P}<0.05,{ }^{\&} \mathrm{P}<0.05$ and ${ }^{\%} \mathrm{P}<0.05$ with comparisons shown by lines. Scale bar, $100 \mu \mathrm{m}$. tMCAO, transient middle cerebral artery occlusion; Ad, adenovirus; HIF-1 $\alpha$, hypoxia-inducible factor-1 $\alpha$; EMP9, erythropoietin mimetic peptide-9.

EMP9, Ad-HIF-1 $\alpha$ significantly decreased the number of apoptotic cells $(\mathrm{P}<0.05$; Fig 2I).

Ad-HIF-1 $\alpha$ suppresses active caspase-3 in neurons. Active caspase- 3 is a cysteine protease involved in the activation cascade of caspases responsible for apoptosis execution (28). A very low expression of active caspase 3 was revealed in the Sham group (Fig. 3A). In comparison, numerous active caspase 3-positive cells were observed in the brain of rats treated with Ad (Fig. 3B). Active caspase 3 expression was downregulated by Ad-HIF-1 $\alpha$ treatment (Fig. 3C). EMP9 treatment did not change the active caspase- 3 expression induced by tMCAO (Fig. 3D). Statistical analysis demonstrated that Ad-HIF-1 $\alpha$ treatment significant decreased active caspase 3 

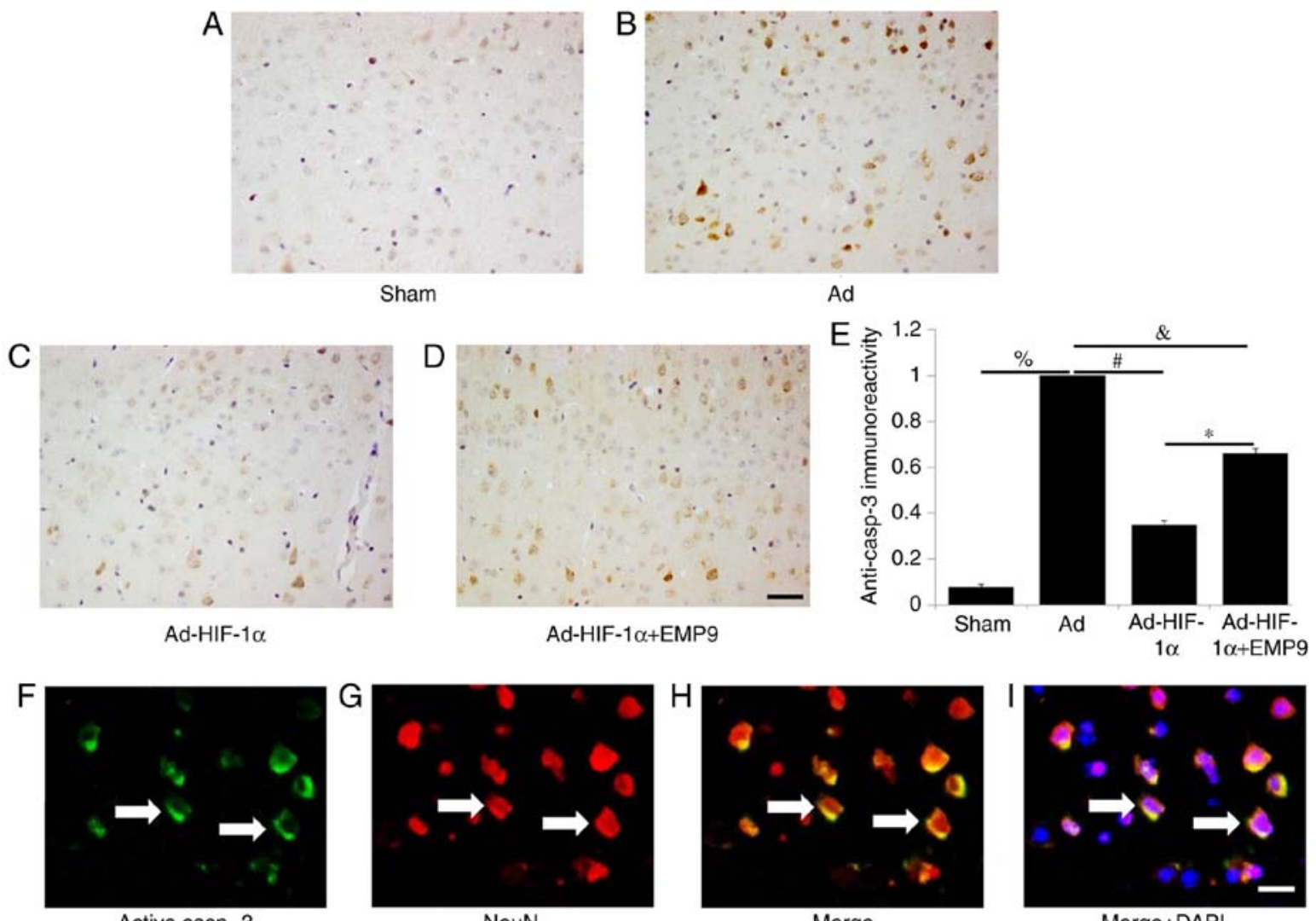

Figure 3. Ad-HIF-1 $\alpha$ treatment suppressed active caspase-3 expression in neurons. Active caspase-3 was detected in (A) the Sham group, (B) the Ad group, (C) the Ad-HIF-1 $\alpha$ and (D) the Ad-HIF-1 $\alpha+$ EMP9 group (scale bar, $50 \mu \mathrm{m}$ ), and then (E) quantified. There were significantly more active caspase 3-positive cells in the brains of rats treated with Ad compared with the Sham group. ${ }^{\circ} \mathrm{P}<0.05,{ }^{*} \mathrm{P}<0.05,{ }^{\circledR} \mathrm{P}<0.05$ and ${ }^{*} \mathrm{P}<0.05$ with comparisons shown by lines. (F) Active caspase 3 and (G) NeuN double-labeled fluorescence staining in Ad-treated rat brains (scale bar, $20 \mu \mathrm{m}$ ). (H) Colocalization of NeuN (red) and active caspase 3 (green) (white arrows; scale bar, $20 \mu \mathrm{m}$ ). (I) Colocalization of NeuN (red), and active casp3 (green) and DAPI (blue) (white arrows; scale bar, $20 \mu \mathrm{m}$ ). Ad, adenovirus; HIF-1 $\alpha$, hypoxia-inducible factor-1 $\alpha$; EMP9, erythropoietin mimetic peptide-9; NeuN, RNA binding fox-1 homolog 3.

expression compared with the Ad group ( $\mathrm{P}<0.05$; Fig. 3E). The present study used double-labeled fluorescence staining to determine whether active caspase-3 (Fig. 3F) was expressed in neurons (Fig. 3G). The results demonstrated that active caspase-3 was expressed in neurons (Fig. $3 \mathrm{H}$ ). Colocalization of active caspase-3 (green), NeuN (red) and DAPI (blue) staining (Fig. 3I) further confirmed the co-expression.

Ad-HIF-1 $\alpha$ upregulated HIF-1 $\alpha$ expression following tMCAO. An extremely low expression of HIF-1 $\alpha$ was observed in the cytoplasm of neurons in the Sham group (Fig. 4A). In brain of rats treated with Ad, HIF-1 $\alpha$ nuclear positive cells demonstrated that tMCAO induces HIF- $1 \alpha$ activation (Fig. 4B). HIF-1 $\alpha$ was expressed in neurons and glia cells. HIF-1 $\alpha$ expression was upregulated by Ad-HIF-1 $\alpha$ treatment (Fig. 4C) and EMP9 treatment did not attenuate the effect (Fig. 4D). Statistical analysis revealed that Ad-HIF-1 $\alpha$ treatment significantly increased HIF-1 $\alpha$ expression compared with the Ad-treated group $(\mathrm{P}<0.05$; Fig. 4E). To further confirm that Ad-HIF-1 $\alpha$ upregulated HIF-1 $\alpha$ expression, HIF-1 $\alpha$ western blot analysis for Ad-treated rats and Ad-HIF-1 $\alpha$-treated rats was performed. The results demonstrated that compared with Ad treatment, HIF-1 $\alpha$ was increased $~ 3.4$ fold by Ad-HIF-1 $\alpha$ treatment $(\mathrm{P}<0.05$; Fig. S1).

The present study used double-labeled fluorescence staining to determine whether HIF-1 $\alpha$ (Fig. 5A) was expressed in neurons (Fig. 5B). The results demonstrated that activated HIF-1 $\alpha$ was observed in neurons (Fig. 5C). Colocalization of active HIF-1 $\alpha$ (red), NeuN (green) and DAPI (blue) staining (Fig. 5D) further confirmed the co-expression. The present study additionally used double-labeled fluorescence staining to determine whether HIF-1 $\alpha$ (Fig. 5E) was expressed in astrocytes (Fig. 5F), which are EPO-producing cells. Activated HIF-1 $\alpha$ was observed in astrocytes (Fig. 5G). It was further confirmed by the colocalization of HIF-1 $\alpha$ (red), GFAP (green) and DAPI (blue) staining (Fig. 5H). In a preliminary experiment, on day 3 rats treated by Ad-HIF-1 $\alpha$ had more activated GFAP-positive astrocytes compared with that in Ad treated rats (data not shown). However on day 7, no significant difference was observed either in the number or the morphology of astrocytes (data not shown).

Ad-HIF-1 $\alpha$ increased EPO expression without changing the expression of EPO-R. To determine the effect of HIF-1 $\alpha$ on the expression of EPO and EPO-R, RT-qPCR and western blot analysis were performed. EPO $\mathrm{mRNA}$ and protein levels in the Ad group were $\sim 1.8$ and $\sim 1.7$ fold, respectively, higher compared with that in the Sham group ( $\mathrm{P}<0.05$; Fig. 6A and D). Compared with Ad treatment, Ad-HIF-1 $\alpha$ treatment increased EPO mRNA levels 2.6 fold (P<0.05; Fig. 6A). Ad-HIF-1 $\alpha$ treatment did not significantly alter EPO-R mRNA expression levels (Fig. 6B). Compared with Ad treatment, Ad-HIF-1 $\alpha$ 

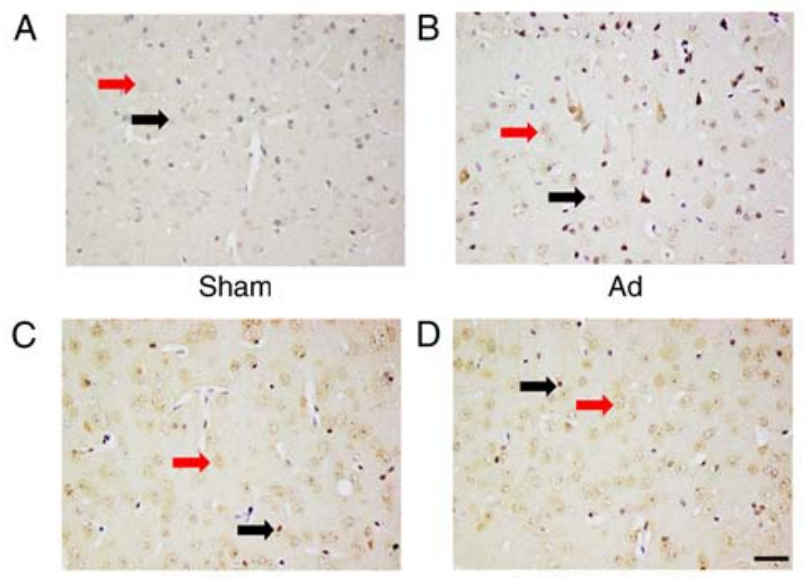

Ad-HIF-1 $\alpha$
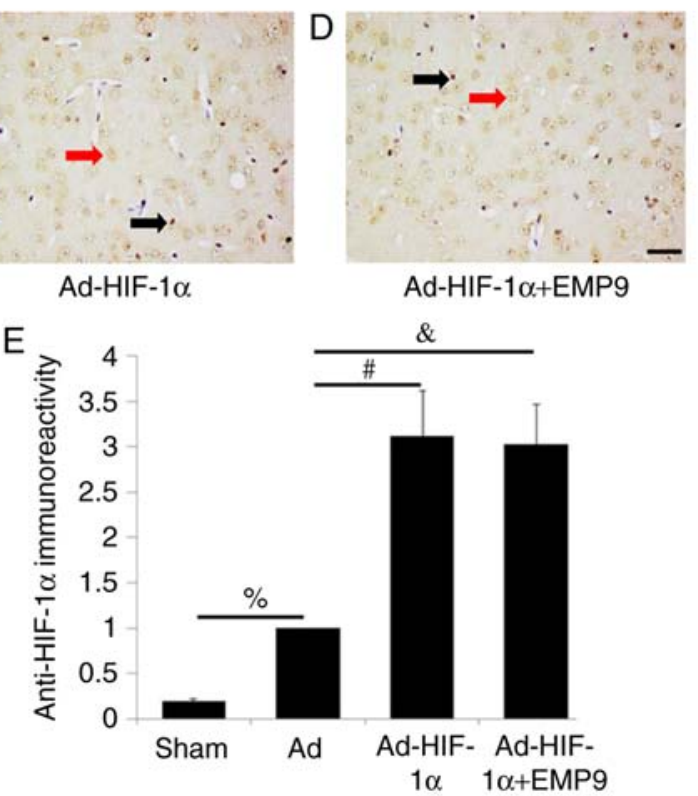

Figure 4. Ad-HIF-1 $\alpha$ treatment enhanced HIF-1 $\alpha$ expression following MCAO. (A) In the Sham group, a very low expression of HIF-1 $\alpha$ was revealed in cytoplasm of neurons. (B) There were some HIF-1 $\alpha$-positive cells in the brains of rats treated with Ad. (C) There were substantially more HIF-1 $\alpha$-positive cells in Ad-HIF-1 $\alpha$-treated rats compared with rats treated with Ad. (D) EMP9 treatment did not change HIF-1 $\alpha$ expression increased by Ad-HIF-1 $\alpha$. (E) Quantified HIF-1 $\alpha$ expression levels. ${ }^{\%} \mathrm{P}<0.05,{ }^{~} \mathrm{P}<0.05$ and ${ }^{\circledR} \mathrm{P}<0.05$ with comparisons shown by lines. Red arrows indicate HIF-1 $\alpha$ expression in neurons. Black arrows indicate HIF-1 $\alpha$ expression in glia cells. Scale bar, $50 \mu \mathrm{m}$. MCAO, middle cerebral artery occlusion; Ad, adenovirus; HIF-1 $\alpha$, hypoxia-inducible factor-1 $\alpha$; EMP9, erythropoietin mimetic peptide-9.

treatment increased EPO protein expression (Fig. 6C). Quantitative analysis revealed that EPO protein levels were upregulated $\sim 2.7$ folds $(\mathrm{P}<0.05$; Fig. 6D). EMP9 treatment did not affect EPO expression increased by HIF-1 $\alpha$ at the protein level (Fig. 6C). Ad-HIF-1 $\alpha$ treatment did not change EPO-R protein expression (Fig. 6C). No significant difference was observed in EPO-R protein expression levels between the different groups (Fig. 6E).

\section{Discussion}

The present study investigated whether HIF-1 $\alpha$ inhibits neuronal apoptosis through upregulating EPO in a rat model of stroke. The results demonstrated that Ad-HIF-1 $\alpha$ treatment resulted in improved neurological functional recovery by decreasing neuronal apoptosis. The results of the present study further proved that the administration of Ad-HIF-1 $\alpha$ upregulated HIF-1 $\alpha$ expression in neurons and astrocytes. Finally, the present study proved that Ad-HIF-1 $\alpha$ treatment upregulated EPO expression, which in turn suppressed activated caspase-3 in neurons. The results presented here provide novel insights into the function of HIF-1 $\alpha$ for treating ischemic stroke.
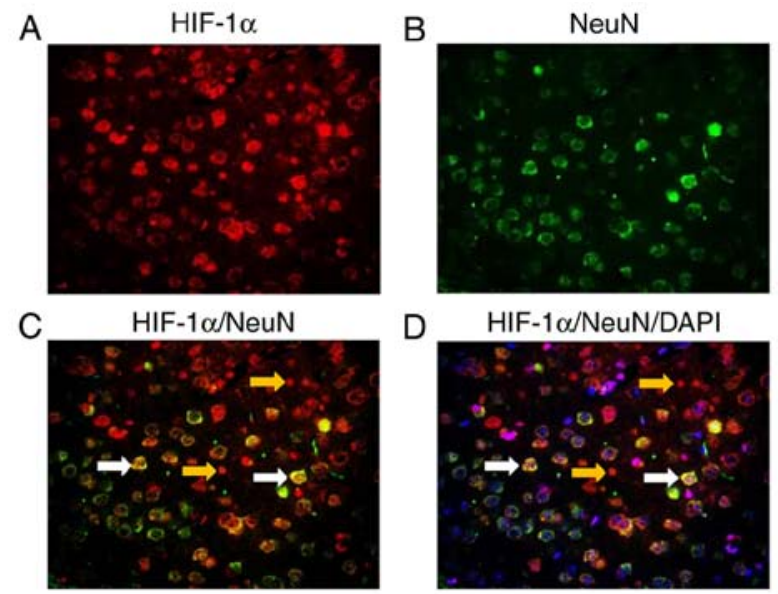

E

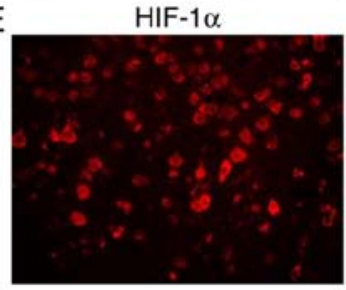

F

GFAP

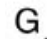

HIF- $1 \alpha /$ GFAP
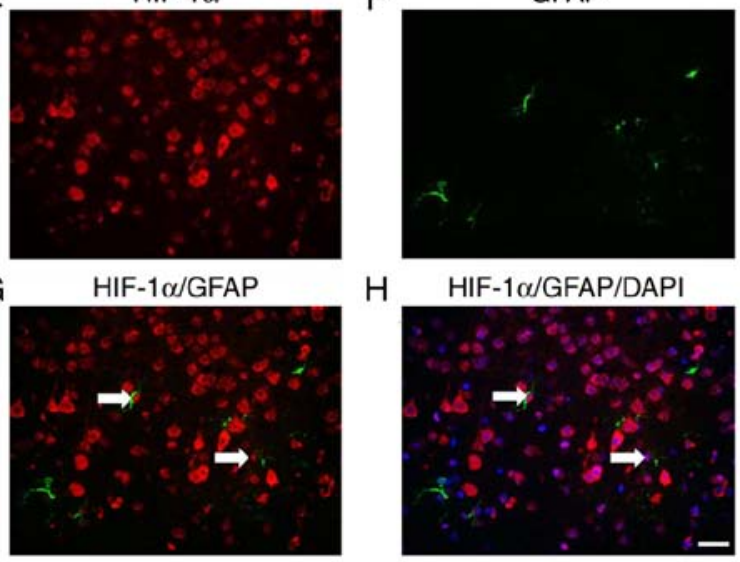

$\mathrm{H}$

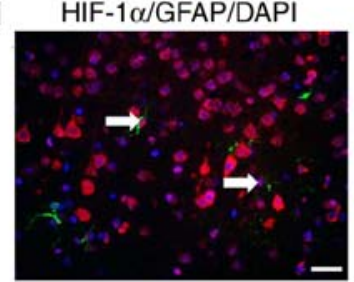

Figure 5. Expression of HIF-1 $\alpha$ in neurons and astrocytes. (A) HIF-1 $\alpha$ and (B) NeuN double-labeled fluorescence staining in Ad-HIF-1 $\alpha$ treated rat brains. (C) Colocalization of HIF-1 $\alpha$ (red) and NeuN (green) (white arrows). (D) Colocalization of HIF-1 $\alpha$ (red), NeuN (green) and DAPI (blue) (white arrows). Yellow arrows indicate cells that were HIF- $1 \alpha$-positive but NeuNnegative. (E) HIF-1 $\alpha$ and (F) GFAP double-labeled fluorescence staining in Ad-HIF-1 $\alpha$ treated rat brains. (G) Colocalization of HIF-1 $\alpha$ (red) and NeuN (green) (white arrows). (H) Colocalization of HIF-1 $\alpha$ (red), NeuN (green) and DAPI (blue) (white arrows). Scale bars: $50 \mu \mathrm{m}$. Ad, adenovirus; HIF-1 $\alpha$, hypoxia-inducible factor-1 $\alpha$; NeuN, RNA binding fox-1 homolog 3; GFAP, glial fibrillary acidic protein.

Stroke is one of the most common causes of adult permanent disability globally, with $\sim 90 \%$ of cases resulting from an ischemic stroke, which is an acute reduction in cerebral blood supply to the brain tissues (29). Reduced oxygen and nutrients following focal ischemic stroke affect a small core of ischemic tissues initially; however, the infarct expands over hours and days, due to a loss of ion homeostasis, excitatory amino acid release, edema and decreased $\mathrm{pH}$, causing cell apoptosis in the surrounding tissues of the brain parenchyma (30). These areas surrounding the ischemic core are defined as the ischemic penumbra, a region where selective neuronal death, hypoxia, protein denaturation and limited diffusion happen at the same time. The penumbra region exists between the normal cerebral blood flow region and infarct core area, and the gene expression is different in various regions at different time points (31). The tissue of the ischemic penumbra is reversible through timely treatment, so that for post-ischemic neuroprotective therapies the strategy is to target the ischemic penumbra for preventing or rescuing the spreading damage from the initial infarct (32). In the present study, it was revealed 

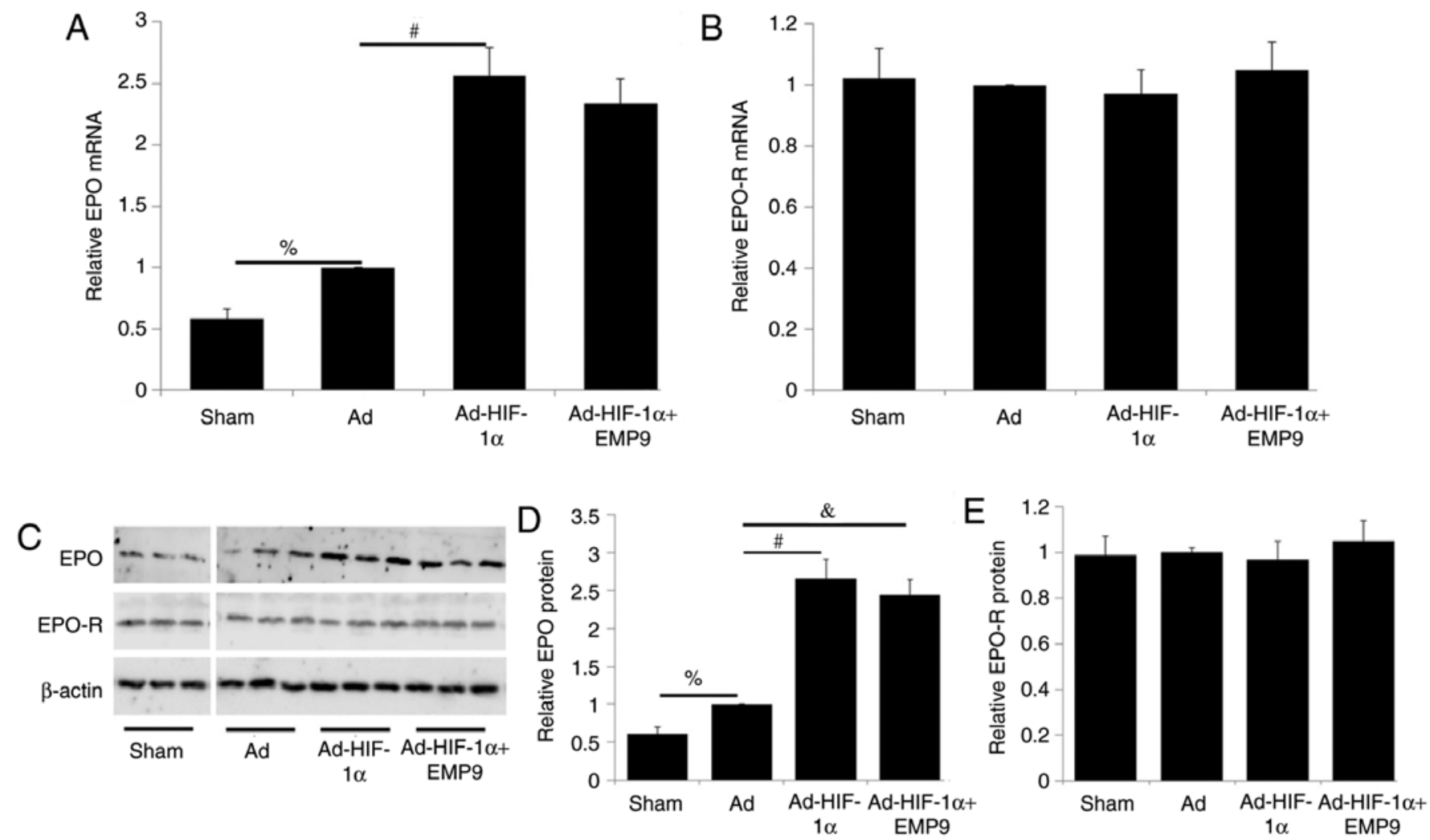

Figure 6. Ad-HIF-1 $\alpha$ treatment upregulated EPO expression without changing EPO-R expression following MCAO. (A) Compared with rats in the Sham group, Ad-treated rats exhibited a significant increase in EPO mRNA levels. Ad-HIF-1 $\alpha$ treatment upregulated EPO mRNA 2.6 fold compared with the Ad-treated group. EMP9 treatment did not affect Ad-HIF-1 $\alpha$ increased EPO mRNA expression. (B) Neither Ad-HIF-1 $\alpha$ treatment nor Ad-HIF-1 $\alpha+E M P 9$ treatment altered EPO-R mRNA expression levels. (C) Western blot analysis was performed for EPO, EPO-R and $\beta$-actin. (D) Quantified EPO protein levels. Ad treatment upregulated EPO protein levels $\sim 1.7$ fold compared with that in the Sham group. Ad-HIF-1 $\alpha$ treatment increased EPO protein levels $\sim 4.9$ fold compared with the Ad group. EMP9 treatment did not affect Ad-HIF-1 $\alpha$-increased EPO protein expression levels. (E) Ad-HIF-1 $\alpha$ treatment and Ad-HIF-1 $\alpha+E M P 9$ treatment did not alter EPO-R protein expression levels. ${ }^{\%} \mathrm{P}<0.05,{ }^{~} \mathrm{P}<0.05$ and ${ }^{\&} \mathrm{P}<0.05$ with comparisons shown by lines. MCAO, middle cerebral artery occlusion; Ad, adenovirus; HIF-1 $\alpha$, hypoxia-inducible factor-1 $\alpha$; EMP9, erythropoietin mimetic peptide-9; EPO, erythropoietin; EPO-R, erythropoietin receptor.

that the administration of Ad-HIF-1 $\alpha$ in the ischemic penumbra subsequent to focal ischemia reduced neuronal apoptosis and behavioral deficits associated with the upregulated expression of HIF-1 $\alpha$ and EPO, a HIF-1-regulated gene.

The ischemic penumbra remains the main target for ischemic stroke treatment, as some tissue in the ischemic penumbra is still salvageable surrounding the core of infarction $(31,32)$. Neurons in the ischemic penumbra are at a high risk for delayed apoptosis caused by the deleterious metabolic processes propagated from the ischemic core, including the loss of ion homeostasis, excitatory amino acid release, inflammation, decreased $\mathrm{pH}$ and edema. Following stroke, the surviving neurons in the ischemic penumbra directly contribute to neurological function recovery (33). HIF-1 $\alpha$, pathologically stimulated in the ischemic region, is of central importance in the response to hypoxia/ischemia, and is crucial for the endogenous protection against low oxygen. However, reports around the pharmacological or genetic alteration of HIF activity to treat cerebral ischemia are controversial. Studies have revealed that HIF-1 $\alpha$ induction decreased neuronal death in the ischemic penumbra (14,34-38). While neuron-specific HIF-1 $\alpha$ knockout increased tissue injury following an ischemic stroke (39), in others, it improved neuronal survival and sensorimotor function $(40,41)$. In these two previous studies, the function of HIF-1 $\alpha$ knockout in the early acute stage
(48 and 72 h) was investigated. Furthermore, HIF-1 $\alpha$ inhibition by 3-(5'-hydroxymethyl-2'-furyl)-1-benzylindazole (YC-1) has also been revealed to promote brain damage following stroke while simultaneously decreasing ischemia-induced vascular leakage (42). In this previous study, the results demonstrated that YC-1 not only inhibited ischemia-induced HIF-1 but also downregulated the HIF-1 downstream genes EPO, VEGF and glucose transporter in neurons and brain endothelial cells. So, it was concluded that YC-1 lacks the potential to function as a cerebral ischemic treatment. Thus, further studies are required to clarify the exact function of HIF-1 and its target genes for stroke outcome and prevention. In the present study, for the first time, an adenovirus was used to overexpress HIF-1 $\alpha$ in the ischemic penumbra. The adenovirus themselves did not affect the HIF-1 downstream genes. The present study demonstrated that Ad-HIF-1 $\alpha$ administration increased HIF-1 $\alpha$ protein expression in neurons and astrocytes. Ad-HIF-1 $\alpha$ treatment also upregulated EPO expression without a significant change in EPO-R expression in the ischemic penumbra. More importantly, the neuroprotection exerted by Ad-HIF-1 $\alpha$ was partially diminished by EMP9, an erythropoietin receptor antagonist, proving that the neuroprotective effect of HIF-1 is mediated by EPO upregulation. Furthermore, the present study demonstrated that EMP9 failed to alter the expression of EPO upregulated by Ad-HIF-1 $\alpha$, which indicates that the HIF-1 $\alpha$ neuroprotective 
effect depends on upregulated EPO functionally binding to its receptor. Finally, the present study demonstrated that Ad-HIF-1 $\alpha$ treatment upregulated EPO expression, which in turn suppresses activated caspase- 3 in neurons.

Caspases may be divided into two groups based on their function in apoptosis (caspase-3, 6, 7, 8 and 9) and inflammation (caspase-1, 4, 5 and 12) (43). Caspase-3, a major executioner caspase in apoptosis, is cleaved and activated by caspase- 8 and caspase-9. The active caspase- 3 enzyme is formed once caspase- 3 is cleaved at an aspartate residue to yield a p12 and a p17 subunit (44). During apoptosis, active caspase-3 degrades multiple cellular proteins and is responsible for DNA fragmentation and morphological changes in cells (41). EPO has been reported to have an anti-apoptosis function (45). The EPO-R is dimerized once it binds to EPO that in turn activates Janus kinase 2 (JAK2), a receptor-associated tyrosine kinase. Activated JAK2 phosphorylates tyrosine residues on the cytosolic domain of the EPO-R, which results in the recruitment of downstream effectors, including growth factor receptor bound protein 2, phosphoinositide-3 kinase (PI3K) and signal transducer and activator of transcription family members $(45,46)$. It has been revealed that EPO prevents PC12 cell injuries by activating the $\mathrm{PI} 3 \mathrm{~K} /$ protein kinase B pathway (47). EPO is also able to enhance the antioxidant capacity by activating the reactive oxygen species neutralizing enzymes, including superoxide dismutase, glutathione peroxidase and catalase (48-50).

In the present study, the neuroprotection by Ad-HIF-1 $\alpha$ was not completely diminished by EMP9, indicating that other HIF-1 $\alpha$-regulated pathways are involved in HIF-1 $\alpha$ mediated neuroprotection. HIF- $1 \alpha$ is a universal molecular master switch, controlling cellular survival, metabolic adaptation and glucose metabolism and transport. More than 30 target genes are known for HIF-1 $\alpha$, including but not limited to EPO, VEGF, insulin-like growth factor 2, glycolytic enzymes and glucose transporter $1(51,52)$. Among them, VEGF is the most important angiogenetic factor in all steps of angiogenesis (53). VEGF participates in angiogenesis via recruiting endothelial cells into a hypoxic and avascular area and stimulating their proliferation. Therefore, VEGF induction, among other proangiogenic factors, in the ischemic area causes an increase in the vascular density and a decrease in the oxygen diffusion distances, resulting in neuronal apoptosis inhibition (53). More studies are required to investigate other mechanisms involved in HIF-1 $\alpha$-mediated neuroprotection.

In the present study, TUNEL staining was used to identify apoptotic cells. It is well known that TUNEL is unable to distinguish apoptosis from necrosis. Furthermore apoptosis and necrosis may coexist in the same tissue. However, in a tMCAO model, necrosis mostly locates in the ischemic core and apoptosis more frequently occurs in the ischemic penumbra (54). In the present study, ischemic penumbra tissue was examined. Therefore, in combination with active caspase- 3 staining, the majority of TUNEL-positive signals represent apoptotic cells.

In conclusion, the present study demonstrated that HIF-1 $\alpha$ attenuates neuronal apoptosis partially through upregulating EPO, which in turn suppresses activated caspase-3 in neurons following cerebral ischemia in rats. The data presented by the present study provides novel insights into the function of HIF-1 $\alpha$ in the treatment of ischemic stroke. Thus, HIF-1 $\alpha$ may be a potential therapeutic target for ischemic stroke.

\section{Acknowledgements}

The authors would like to thank Mr. Arvand Asghari from the University of Houston (Houston, TX, USA) for carefully editing the English of the manuscript.

\section{Funding}

The present study was supported by the Health and Family Planning Commission of Guizhou (grant nos. 2014-41 and gzwjkj 2014-1-055), the Science and Technology Foundation of Guizhou (grant no. 2007-2096) and the Special Governor's Fund for Outstanding Technological and Educational Talents in Guizhou (grant no. 2006-60).

\section{Availability of data and materials}

The data used and/or analyzed in this study are available from the corresponding author with reasonable request.

\section{Authors' contributions}

$\mathrm{JL}$ and TT wrote the manuscript, and contributed to the acquisition, analysis and interpretation of the data. JX, ZL, ZZ and MJ collected, analyzed and interpreted the data. JL, TT, JX and ZL contributed to the conception and design of the present study. All authors read and approved the final manuscript.

\section{Ethics approval and consent to participate}

The animal studies were ethically approved by the local Animal Experimentation Ethics Committee (Guizhou Provincial People's Hospital, Guizhou, China) for animal experimentation.

\section{Patient consent for publication}

Not applicable.

\section{Competing interests}

The authors declare that they have no competing interests.

\section{References}

1. Ng M, Fleming T, Robinson M, Thomson B, Graetz N, Margono C, Mullany EC, Biryukov S, Abbafati C, Abera SF, et al: Global, regional, and national prevalence of overweight and obesity in children and adults during 1980-2013: A systematic analysis for the Global Burden of Disease Study 2013. Lancet 384: 766-781, 2014.

2. GBD 2016 Causes of Death Collaborators: Global, regional, and national age-sex specific mortality for 264 causes of death, 1980-2016: A systematic analysis for the Global Burden of Disease Study 2016. Lancet 390: 1151-210, 2017.

3. Yang G, Wang Y, Zeng Y, Gao GF, Liang X, Zhou M, Wan X, Yu S, Jiang Y, Naghavi M, et al: Rapid health transition in China, 1990-2010: Findings from the global burden of disease study 2010. Lancet 381: 1987-2015, 2013.

4. Liu L, Wang D, Wong KS and Wang Y: Stroke and stroke care in China: Huge burden, significant workload, and a national priority. Stroke 42: 3651-3654, 2011.

5. Wang W, Jiang B, Sun H, Ru X, Sun D, Wang L, Wang L, Jiang Y, Li Y, Wang Y, et al: Prevalence, incidence, and mortality of stroke in China: Results from a nationwide population-based survey of 480687 adults. Circulation 135: 759-771, 2017. 
6. Wang YJ, Zhang SM, Zhang L, Wang CX, Dong Q, Gao S, Huang RX, Huang YN, Lv CZ, Liu M, et al: Chinese guidelines for the secondary prevention of ischemic stroke and transient ischemic attack 2010. CNS Neurosci Ther 18: 93-101, 2012.

7. Hacke W, Kaste M, Bluhmki E, Brozman M, Davalos A Guidetti D, Larrue V, Lees KR, Medeghri Z, Machnig T, et al: Thrombolysis with alteplase 3 to 4.5 hours after acute ischemic stroke. N Engl J Med 359: 1317-1329, 2008.

8. Xydas T, Georgantopoulos C, Bethanis D and Sarris M: Thrombolysis for acute ischemic stroke: A new paradigm. Hosp Chron 7: 77-80, 2012.

9. National 'Ninth Five-Year Plan' Research Group; Chen Q Intravenous thrombolysis with urokinase for acute cerebral infarctions. Chin J Neurol 35: 210-213, 2002 (In Chinese).

10. Semenza GL: Targeting HIF-1 for cancer therapy. Nat Rev Cancer 3: 721-732, 2003

11. Semenza GL: Hypoxia-inducible factor 1: Oxygen homeostasis and disease pathophysiology. Trends Mol Med 7: 345-350, 2001.

12. Ivan M, Kondo K, Yang H, Kim W, Valiando J, Ohh M, Salic A, Asara JM, Lane WS and Kaelin WG Jr: HIFalpha targeted for VHL-mediated destruction by proline hydroxylation: Implications for O2 sensing. Science 292: 464-468, 2001.

13. Abe H, Semba $\mathrm{H}$ and Takeda $\mathrm{N}$ : The roles of hypoxia signaling in the pathogenesis of cardiovascular diseases. J Atheroscler Thromb 24: 884-894, 2017.

14. Feng $\mathrm{Y}$ and Bhatt AJ: Corticosteroid responses following hypoxic preconditioning provide neuroprotection against subsequent hypoxic-ischemic brain injury in the newborn rats. Int J Dev Neurosci 44: 6-13, 2015.

15. Chen J, Yang Y, Shen L, Ding W, Chen X, Wu E, Cai K and Wang G: Hypoxic preconditioning augments the therapeutic efficacy of bone marrow stromal cells in a rat ischemic stroke model. Cell Mol Neurobiol 37: 1115-1129, 2017.

16. Jacobs K, Shoemaker C, Rudersdorf R, Neill SD, Kaufman RJ, Mufson A, Seehra J, Jones SS, Hewick R and Fritsch EF: Isolation and characterization of genomic and cDNA clones of human erythropoietin. Nature 313: 806-810, 1985.

17. Chikuma M, Masuda S, Kobayashi T, Nagao M and Sasaki R Tissue-specific regulation of erythropoietin production in the murine kidney, brain, and uterus. Am J Physiol Endocrinol Metab 279: E1242-E1248, 2000.

18. Yu X, Shacka JJ, Eells JB, Suarez-Quian C, Przygodzki RM, Beleslin-Cokic B, Lin CS, Nikodem VM, Hempstead B, Flanders KC, et al: Erythropoietin receptor signalling is required for normal brain development. Development 129: 505-516, 2002.

19. Iwai M, Stetler RA, Xing J, Hu X, Gao Y, Zhang W, Chen J and Cao G: Enhanced oligodendrogenesis and recovery of neurological function by erythropoietin after neonatal hypoxic/ischemic brain injury. Stroke 41: 1032-1037, 2010.

20. Wu SH, Lu IC, Lee SS, Kwan AL, Chai CY and Huang SH: Erythropoietin attenuates motor neuron programmed cell death in a burn animal model. PLoS One 13, e0190039, 2018.

21. Wang P, Lu Y, Han D, Wang P, Ren L, Bi J and Liang J: Neuroprotection by nicotinamide mononucleotide adenylyltransferase 1 with involvement of autophagy in an aged rat model of transient cerebral ischemia and reperfusion. Brain Res 1723: 146391, 2019.

22. Saadoun S, Waters P, Bell BA, Vincent A, Verkman AS and Papadopoulos MC: Intra-cerebral injection of neuromyelitis optica immunoglobulin $\mathrm{G}$ and human complement produces neuromyelitis optica lesions in mice. Brain 133: 349-361, 2010.

23. Chen T, Yu Y, Tang LJ, Kong L, Zhang CH, Chu HY, Yin LW and Ma HY: Neural stem cells over-expressing brain-derived neurotrophic factor promote neuronal survival and cytoskeletal protein expression in traumatic brain injury sites. Neural Regen Res 12: 433-439, 2017.

24. Wu W, Chen X, Hu C, Li J, Yu Z and Cai W: Transplantation of neural stem cells expressing hypoxia-inducible factor-1alpha (HIF-1alpha) improves behavioral recovery in a rat stroke model. J Clin Neurosci 17: 92-95, 2010.

25. Yang ML, Tao T, Xu J, Liu Z and Xu D: Antiapoptotic effect of gene therapy with recombinant adenovirus vector containing hypoxia-inducible factor- $1 \alpha$ after cerebral ischemia and reperfusion in rats. Chin Med J (Engl) 130: 1700-1706, 2017.

26. Lin YW and Hsieh CL: Electroacupuncture at Baihui acupoint (GV20) reverses behavior deficit and long-term potentiation through N-methyl-d-aspartate and transient receptor potential vanilloid subtype 1 receptors in middle cerebral artery occlusion rats. J Integr Neurosci 9: 269-282, 2010.

27. Livak KJ and Schmittgen TD: Analysis of relative gene expression data using real-time quantitative PCR and the 2(-Delta Delta C(T)) method. Methods 25: 402-408, 2001.
28. Snigdha S, Smith ED, Prieto GA and Cotman CW: Caspase-3 activation as a bifurcation point between plasticity and cell death. Neurosci Bull 28: 14-24, 2012.

29. Li Y, Huang L, Ma Q, Concepcion KR, Song MA, Zhang P, Fu Y, Xiao D and Zhang L: Repression of the glucocorticoid receptor aggravates acute ischemic brain injuries in adult mice. Int $\mathrm{J}$ Mol Sci 19: E2428, 2018.

30. Paciaroni M, Caso V and Agnelli G: The concept of ischemic penumbra in acute stroke and therapeutic opportunities. Eur Neurol 61: 321-330, 2009.

31. Rosso C and Samson Y: The ischemic penumbra: The location rather than the volume of recovery determines outcome. Curr Opin Neurol 27: 35-41, 2014

32. Ran YC, Zhu M, Li SJ, Zhang ZX, Wang X, Zhang Y and Cheng JL: Related research and recent progress of ischemic penumbra. World Neurosurg 116: 5-13, 2018.

33. Murphy TH and Corbett D: Plasticity during stroke recovery: From synapse to behaviour. Nat Rev Neurosci 10: 861-872, 2009.

34. Reischl S, Li L, Walkinshaw G, Flippin LA, Marti HH and Kunze R: Inhibition of HIF prolyl-4-hydroxylases by FG-4497 reduces brain tissue injury and edema formation during ischemic stroke. PLoS One 9: e84767, 2014.

35. Ogle ME, Gu X, Espinera AR and Wei L: Inhibition of prolyl hydroxylases by dimethyloxaloylglycine after stroke reduces ischemic brain injury and requires hypoxia inducible factor- $1 \alpha$. Neurobiol Dis 45: 733-742, 2012.

36. Li C, Zhang B, Zhu Y, Li Y, Liu P, Gao B, Tian S, Du L and Bai Y: Post-stroke constraint-induced movement therapy increases functional recovery, angiogenesis, and neurogenesis with enhanced expression of HIF-1 $\alpha$ and VEGF. Curr Neurovasc Res 14: 368-377, 2017.

37. Wang H, Niu F, Fan W, Shi J, Zhang J and Li B: Modulating effects of preconditioning exercise in the expression of ET-1 and BNP via HIF-1 $\alpha$ in ischemically injured brain. Metab Brain Dis 34: 1299-1311, 2019.

38. Gao Y, Yin H, Zhang Y, Dong Y, Yang F, Wu X and Liu H: Dexmedetomidine protects hippocampal neurons against hypoxia/reoxygenation-induced apoptosis through activation HIF-1 $\alpha /$ p53 signaling. Life Sci 232: 116611, 2019.

39. Baranova O, Miranda LF, Pichiule P, Dragatsis I, Johnson RS and Chavez JC: Neuron-specific inactivation of the hypoxia inducible factor 1 alpha increases brain injury in a mouse model of transient focal cerebral ischemia. J Neurosci 27: 6320-6332, 2007.

40. Barteczek P, Li L, Ernst AS, Böhler LI, Marti HH and Kunze R: Neuronal HIF- $1 \alpha$ and HIF- $2 \alpha$ deficiency improves neuronal survival and sensorimotor function in the early acute phase after ischemic stroke. J Cereb Blood Flow Metab 37: 291-306, 2017.

41. Helton R, Cui J, Scheel JR, Ellison JA, Ames C, Gibson C, Blouw B, Ouyang L, Dragatsis I, Zeitlin S, et al: Brain-specific knock-out of hypoxia-inducible factor-1 alpha reduces rather than increases hypoxic-ischemic damage. J Neurosci 25: 4099-4107, 2005.

42. Yan J, Zhou B, Taheri S and Shi H: Differential effects of HIF-1 inhibition by $\mathrm{YC}-1$ on the overall outcome and blood-brain barrier damage in a rat model of ischemic stroke. PLoS One 6: e27798, 2011.

43. McIlwain DR, Berger T and Mak TW: Caspase functions in cell death and disease. Cold Spring Harb Perspect Boil 5: a008656, 2013.

44. O'Donovan N, Crown J, Stunell H, Hill AD, McDermott E, O'Higgins $\mathrm{N}$ and Duffy MJ: Caspase 3 in breast cancer. Clin Cancer Res 9: 738-742, 2003

45. Digicaylioglu M and Lipton SA: Erythropoietin-mediated neuroprotection involves cross-talk between Jak2 and NF-kappaB signalling cascades. Nature 412: 641-647, 2001.

46. Bouscary D, Pene F, Claessens YE, Muller O, Chrétien S, Fontenay-Roupie M, Gisselbrecht S, Mayeux P and Lacombe C: Critical role for PI 3-kinase in the control of erythropoietininduced erythroid progenitor proliferation. Blood 101: 3436-3443, 2003.

47. Haq R, Halupa A, Beattie BK, Mason JM, Zanke BW and Barber DL: Regulation of erythropoietin-induced STAT serine phosphorylation by distinct mitogen-activated protein kinases. J Biol Chem 277: 17359-17366, 2002.

48. Zhi-Kun S, Hong-Qi Y, Zhi-Quan W, Jing P, Zhen H and Sheng-Di C: Erythropoietin prevents PC12 cells from beta-amyloid-induced apoptosis via PI3K/Akt pathway. Transl Neurodegener 1: 7, 2012. 
49. Fan X, Heijnen CJ, van der KOOIJ MA, Groenendaal F and van Bel F: Beneficial effect of erythropoietin on sensorimotor function and white matter after hypoxia-ischemia in neonatal mice. Pediatr Res 69: 56-61, 2011.

50. Wang Y, Zhang H, Liu Y, Li P, Cao Z and Cao Y: Erythropoietin (EPO) protects against high glucose-induced apoptosis in retinal ganglional cells. Cell Biochem Biophys 71: 749-755, 2015

51. Cheng L, Yu H, Yan N, Lai K and Xiang M: Hypoxia-inducible factor-1 $\alpha$ target genes contribute to retinal neuroprotection. Front Cell Neurosci 11: 20, 2017.

52. Wang L, Zhao RP, Song XY and Wu WF: Targeting ER $\beta$ in macrophage reduces crown-like structures in adipose tissue by inhibiting osteopontin and HIF-1 $\alpha$. Sci Rep 9: 15762, 2019.
53. Lee SH, Jeong D, Han YS and Baek MJ: Pivotal role of vascular endothelial growth factor pathway in tumor angiogenesis. Ann Surg Treat Res 89: 1-8, 2015

54. Charriaut-Marlangue C, Margaill I, Represa A, Popovici T, Plotkine $\mathrm{M}$ and Ben-Ari Y: Apoptosis and necrosis after reversible focal ischemia: An in situ DNA fragmentation analysis. J Cereb Blood Flow Metab 16: 186-194, 1996.

This work is licensed under a Creative Commons Attribution-NonCommercial-NoDerivatives 4.0 International (CC BY-NC-ND 4.0) License. 